Check for updates

Cite this: RSC Adv., 2017, 7, 32703

Received 8th May 2017

Accepted 20th June 2017

DOI: $10.1039 / \mathrm{c} 7 \mathrm{ra05193j}$

rsc.li/rsc-advances

\section{Improved thermoelectric power factor and conversion efficiency of perovskite barium stannate $\uparrow$}

\begin{abstract}
Jun Li, (D) ab Zuju Ma, (D) ${ }^{a}$ Rongjian Sa ${ }^{a}$ and Kechen Wu*a
In the pursuit of high thermoelectric conversion efficiency, both high operating temperature and high figure of merit $Z T$ are desirable. Thermoelectric materials used in high-temperature power generation are still in great demand. Here we demonstrate the $n$-type doped barium stannate $\left(\mathrm{BaSnO}_{3}\right)$ behaves as a robust candidate, as a high-temperature thermoelectric material, due to its ultrahigh power factor and excellent structural and chemical stability. A systematic calculation focused on the geometric, electronic and thermoelectric transport properties of $\mathrm{BaSnO}_{3}$ is performed by using density functional theory combined with Boltzmann transport theory. It can be noticed that the electrical conductivity of $\mathrm{BaSnO}_{3}$ is improved dramatically when it is $n$-type doped, resulting from the small effective mass and extraordinary high mobility. The power factor maximum reaches $1.5 \times 10^{-3} \mathrm{~W} \mathrm{~m}^{-1} \mathrm{~K}^{-2}$ at $1200 \mathrm{~K}$ with the optimal carrier concentration $1.6 \times 10^{19} \mathrm{~cm}^{-3}$, which suggests the great potential of $\mathrm{BaSnO}_{3}$ as an $\mathrm{n}$-type high temperature thermoelectric material.
\end{abstract}

\section{Introduction}

Thermoelectric materials, which can directly convert heat to electricity, are of great interest in waste heat recovery, solid-state cooling and so on. The performance of thermoelectric materials is evaluated through the dimensionless figure of merit $Z T, Z T=$ $\left(S^{2} \sigma / \kappa\right) T$, where $S, \sigma, \kappa$ and $T$ are the Seebeck coefficient, the electrical conductivity, the thermal conductivity, and the absolute temperature, respectively. $\kappa$ consists of lattice $\left(\kappa_{1}\right)$ and electronic $\left(\kappa_{\mathrm{e}}\right)$ contributions. A high $Z T$ value requires high power factor $\left(S^{2} \sigma\right)$ and low thermal conductivity $(\kappa)$. In recent years, many efforts have been made with regard to reducing the thermal conductivity, such as by nanostructuring or introducing layered structures. ${ }^{1,2}$ The strategies to maximize the power factor include optimal doping and band engineering..$^{3-6}$ However, it should be noted that optimizing all of features of thermoelectric coefficients at the same time is a challenging task due to their interdependent relationships.

Apart from the $Z T$ value being used to evaluate the thermoelectric properties of a material, in a thermoelectric device, the conversion efficiency $(\eta)$ is directly used to assess the performance, which is characterized by $Z T$ and the Carnot efficiency $\eta_{\mathrm{c}}$. A high Carnot limit, $\eta_{\mathrm{c}}=\left(T_{\mathrm{h}}-T_{\mathrm{c}}\right) / T_{\mathrm{c}}$, requires a large

${ }^{a}$ State Key Laboratory of Structural Chemistry, Fujian Institute of Research on the Structure of Matter, Chinese Academy of Sciences, Fuzhou 350002, People's Republic of China. E-mail: wkc@fjirsm.ac.cn; Fax: +86-591-83792932; Tel: +86-591-83792600 ${ }^{b}$ University of Chinese Academy of Sciences, Beijing 100049, People's Republic of China $\dagger$ Electronic supplementary information (ESI) available. See DOI: $10.1039 / \mathrm{c} 7 \mathrm{ra05193j}$ temperature difference between the hot side $\left(T_{\mathrm{h}}\right)$ and cold side $\left(T_{\mathrm{c}}\right)$ of the thermoelectric device. Thus, high temperature thermoelectric materials with good transport properties are highly desirable for power generating $>1000 \mathrm{~K}$. Oxide-based semiconductors are regarded as the candidates for high-temperature thermoelectric applications due to their thermal and chemical stability at high temperature. ${ }^{-9}$ The performance of the traditional oxide thermoelectric materials, such as p-type $\mathrm{Na}_{x}$ $\mathrm{CoO}_{2},{ }^{10,11} \mathrm{Ca}_{3} \mathrm{Co}_{4} \mathrm{O}_{9},{ }^{12,13} \mathrm{BiCuSeO}^{14,15}$ and n-type $\mathrm{SrTiO}_{3},{ }^{16,17}$ $\mathrm{ZnO}^{18}$ can be enhanced by heavy-element doping, band engineering and nanostructuring. Nevertheless, only the p-type layer structured oxide thermoelectrics $\mathrm{Na}_{x} \mathrm{CoO}_{2}$ and $\mathrm{BiCuSeO}$ exhibit high figures of merit approaching to $1,^{10,15}$ which is closely related to their low $\kappa . \mathrm{SrTiO}_{3}$ as a n-type solid thermoelectric material has been widely studied for many years due to the large Seebeck coefficient but limited by the low carrier mobility and high thermal conductivity. ${ }^{19}$ Therefore, exploring efficient ways to improve their electrical performance with stable low thermal conductivity is a promising strategy for oxide-based thermoelectric materials.

Fortunately, the carrier mobility and electrical conductivity of n-type doped $\mathrm{BaSnO}_{3}$ can be dramatically enhanced due to the small effective mass $\left(m^{*}\right){ }^{20-23} \mathrm{BaSnO}_{3}$, a typical perovskitetype compound, behaves as an excellent high-mobility electron transport material composed of abundant elements. The sample of $\mathrm{BaSnO}_{3}$ doped with a few percent La has been reported of an unusually high electron mobility of $320 \mathrm{~cm}^{2} \mathrm{~V}^{-1}$ $\mathrm{s}^{-1}$, which is the highest value reported in perovskite oxides. ${ }^{20,21}$ The ultrahigh carrier mobility is ascribed to the small electron effective mass $m^{*}\left(0.06-0.21 m_{\mathrm{e}}\right),{ }^{23-25}$ resulting from the light 
conduction band. The electrical conductivity is remarkably enhanced up to $10^{5} \mathrm{~S} \mathrm{~m}^{-1}$ with the carrier concentration of $10^{20}$ $\mathrm{cm}^{-3}$, showing a metal-like behavior. ${ }^{26}$ Moreover, $\mathrm{BaSnO}_{3}$ exhibits high thermal stability at high temperature exceed 1273 $\mathrm{K}$, which is seven orders of magnitude more thermally stable in terms of the oxygen diffusion constant compared with $\mathrm{SrTiO}_{3}{ }^{27,28}$ The thermal conductivity of pure $\mathrm{BaSnO}_{3}$ is $9.68 \mathrm{~W}$ $\mathrm{m}^{-1} \mathrm{~K}^{-1}$ at room temperature, ${ }^{29}$ which is smaller than the good oxide-based thermoelectric material $\mathrm{SrTiO}_{3}\left(12 \mathrm{~W} \mathrm{~m}^{-1} \mathrm{~K}^{-1}\right){ }^{30}$ but higher than that of the layered structure, such as $\mathrm{NaCo}_{2} \mathrm{O}_{4}$ $\left(2 \mathrm{~W} \mathrm{~m}^{-1} \mathrm{~K}^{-1}\right)$, BiCuSeO $\left(\sim 0.5 \mathrm{~W} \mathrm{~m}^{-1} \mathrm{~K}^{-1}\right){ }^{31,32}$ But it is in negative correlation with temperature and decreases to $3.2 \mathrm{~W}$ $\mathrm{m}^{-1} \mathrm{~K}^{-1}$ at $1262 \mathrm{~K} .^{29}$ Furthermore, for the doped samples, dopants not only supply carriers to increase the electrical conductivity and optimize the power factor, but also deduce point defect scattering to suppress the lattice thermal conductivity. All these factors suggest the great potential of $\mathrm{BaSnO}_{3}$ as a high temperature thermoelectric material by tuning carrier concentration.

In this work, we systematically investigated the geometric, electronic and thermoelectric properties of $\mathrm{BaSnO}_{3}$ by employing the first principle calculations combined with Boltzmann transport theory. A comparison of the thermoelectric transport properties between n-type and p-type doped samples reveals that the n-type electrical conductivity is several order of magnitude higher than that of p-type one. The ultra-high n-type $\mathrm{PF}, 1.5 \times 10^{-3} \mathrm{~W} \mathrm{~m}^{-1} \mathrm{~K}^{-2}$, is observed at $1200 \mathrm{~K}$ with carrier concentration of $1.6 \times 10^{19} \mathrm{~cm}^{-3}$. This PF value is significantly higher than the highest value is standout among the oxidebased thermoelectric materials and even is comparable to those state-of-the-art thermoelectric materials such as $\mathrm{Bi}_{2} \mathrm{Te}_{3}$ and SnSe. ${ }^{33,34}$

\section{Computational details}

The calculation of geometry optimization and electronic structure of $\mathrm{BaSnO}_{3}$ is performed using the density functional theory (DFT) with project augmented wave (PAW) $)^{35}$ potentials as implemented in the Vienna Ab initio Simulation Package (VASP) code. $^{36}$ For the exchange-correlation potential, we use the standard Heyd-Scuseria-Ernzerhof (HSE06) hybrid functional ${ }^{37}$ to obtain exact band gap. The kinetic energy cutoff of wave functions of $600 \mathrm{eV}$ and a Monkhorst-Pack $k$-mesh of $6 \times 6 \times 6$ are adopted to sample the Inreducible Brillouin Zone (IBZ) in the structure relaxation, with the energy convergence threshold set as $10^{-4} \mathrm{eV}$. Both the cell shape and volume are fully optimized and all atoms are allowed to relax until the maximal Hellmann-Feynman force acting on each atom is less than $0.001 \mathrm{eV}^{-1}$. The denser $k$-point mesh $15 \times 15 \times 15,19 \times 19 \times$ 19 and $21 \times 21 \times 21$ are test respectively to guarantee the convergence. Finally, a Monkhorst-Pack $k$-point mesh of $19 \times$ $19 \times 19$ has been used to calculate the transport coefficients of $\mathrm{BaSnO}_{3}$.

The electronic transport properties are obtained utilizing the semi-classical Boltzmann transport theory as implemented in the BoltzTrap code.$^{38}$ As the band structure is calculated at zero temperature, the effect of temperature $T$ and Fermi level $\mu$ are introduced by using the broadening of Fermi distribution. In the phonon calculation, density functional perturbation theory with an atomic displacement of $0.005 \AA$ is employed to determine the harmonic interatomic force constants (IFCs). Phonon frequencies are obtained from IFCs by using Phonopy package. ${ }^{39} \mathrm{~A} 3 \times 3 \times 3$ supercell of primitive cell containing 135 atoms with $6 \times 6 \times 6 \mathrm{k}$-point mesh is used to calculate harmonic and anharmonic IFCs. Convergence tests with systems composed of up to $4 \times 4 \times 4$ unit cells showed no significant changes in phonon dispersion. The interactions up to third-nearest neighbors are considered when calculating the third order anharmonic IFCs. The lattice thermal conductivity is calculated by solving the phonon Boltzmann transport equation with the relaxation time approximation, as implemented in ShengBTE code. ${ }^{40}$

\section{Results and discussion}

\section{Geometrical and electronic structure}

$\mathrm{BaSnO}_{3}$ has an ideal cubic perovskite structure with space group $P m \overline{3} m$ (no. 221) as shown in Fig. 1(a). After full relaxation, the lattice parameter $a=4.115 \AA$, which is in excellent agreement with experimental value ( $4.12 \AA) .{ }^{41}$ Fig. 1 (b) shows the Brillouin zone of cubic $\mathrm{BaSnO}_{3}$. The thermoelectric transport properties are closely related to the electronic states near the Fermi level. As shown in Fig. 1(c), $\mathrm{BaSnO}_{3}$ is an indirect semiconductor, with the conduction band minimum (CBM) and the valence band maximum (VBM) locating at $T$ and $R$ point respectively. The band gap calculated by HSE06 method is $3.0 \mathrm{eV}$, which is in excellent agreement with the experimental values $(3.1 \mathrm{eV}$ and $3.4 \mathrm{eV})^{42,43}$ and more exact than the previous calculated values based on LDA $(0.79 \mathrm{eV})^{44}$ and GGA $(1.4 \mathrm{eV})$ approximation. ${ }^{45}$ $\mathrm{BaSnO}_{3}$ possesses a large conduction band dispersion at $\Gamma$ point, which originates from $\mathrm{Sn} 5 \mathrm{~s}-\mathrm{O} 2 \mathrm{p}$ antibonding orbital interactions, suggesting the small electron effective mass. By fitting the band structure around CBM using the equation, $m^{*}=$ $\hbar^{2} /\left(d^{2} E / d k^{2}\right)$, we calculated $m^{*}$ of $\mathrm{BaSnO}_{3} 0.26 m_{\mathrm{e}}$. Moreover, the large dielectric constant contributes to remarkable low scattering rates. ${ }^{46}$ According to the relationship of $\mu=e \tau / m^{*}$, high
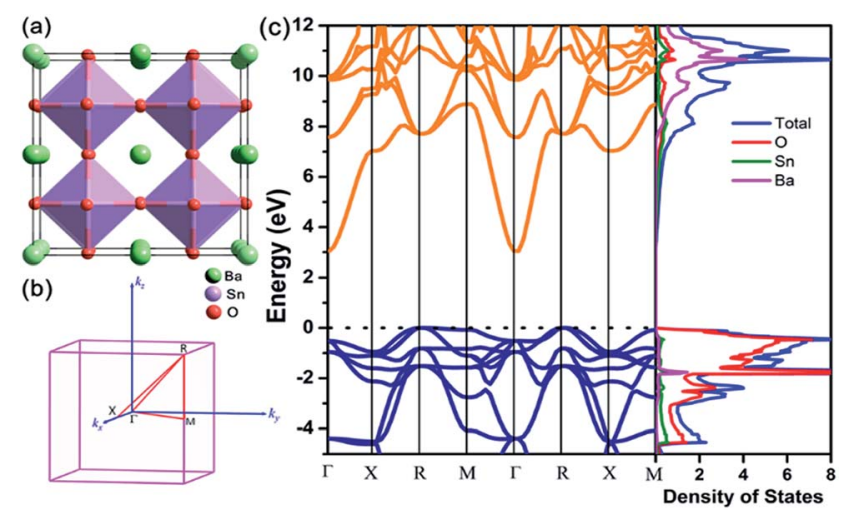

Fig. 1 (a) The optimized crystal structure and (b) Brillouin zone of cubic $\mathrm{BaSnO}_{3}$. (c) Electronic band structure and corresponding density of states based on HSEO6 functional. The Fermi level is set at $0 \mathrm{eV}$. 
mobility for n-type doped can be realized with small electron effective mass. Perovskites are favorable for attempts to introduce carriers through substitutional doping through substitution onto the Ba or Sn site. The extrinsic dopants introduce shallow donor states to the bottom of conduction band and move the Fermi level $\left(E_{\mathrm{f}}\right)$ away from the valance band, which are the source of high conductivity. In contrast, the flat valance band with heavy density-of-states effective mass indicates the high Seebeck coefficient for p-type $\mathrm{BaSnO}_{3}$. To improve the thermoelectric performance of $\mathrm{BaSnO}_{3}$, it is essential to balance both electrical conductivity and Seebeck coefficient.

\section{Thermoelectric properties}

By means of the semi-classical Boltzmann transport theory and invoking the parameters from the calculated electronic structure, the most important thermoelectric properties from electronic contribution of $\mathrm{BaSnO}_{3}$ can be obtained and presented in Fig. 2 . The Seebeck coefficient of $\mathrm{n}$ - and p-type doped $\mathrm{BaSnO}_{3}$ as a function of carrier concentration at different temperature is shown in Fig. 2(a) and (b). The $S$ decreases monotonically with increasing carrier concentration for both $\mathrm{n}$ - and p-type doped sample while increases gradually with the temperature rising. As aforementioned, the flat valance band with heavy density-ofstates effective mass combined with the high valance DOS peak contributes to the larger Seebeck value of p-type doped $\mathrm{BaSnO}_{3}$, comparing to the n-type doped sample. For example, at the same magnitude of hole and electron concentration, $1 \times 10^{19} \mathrm{~cm}^{-3}$, the $S$ of p-type doping sample is about four times higher than that of n-type doping one at $1200 \mathrm{~K}$. Nevertheless, the $S$ of the two cases possesses similar value at their respective optimal carrier concentration of $\sim 10^{21} \mathrm{~cm}^{-3}$ (p-type) and $\sim 10^{19} \mathrm{~cm}^{-3}$ (n-type) for maximal power factor. With the temperature rising, the susceptibility of Seebeck coefficient to temperature is degenerate. It comes very close to the two curves of $1000 \mathrm{~K}$ and $1200 \mathrm{~K}$ for the ptype doped sample.

In fact, only the ratio of electrical conductivity to relaxation time $(\sigma / \tau)$ is obtained directly from the BoltzTrap code. The value of $\sigma / \tau$ for n-type and p-type doped $\mathrm{BaSnO}_{3}$ as functions of temperature and carrier concentration are displayed in Fig. 2(c) and (d). It can be found that $\sigma / \tau$ is almost temperature independent when the temperature increased from $300 \mathrm{~K}$ to $1200 \mathrm{~K}$, in accordance with the temperature independence of carrier mobility. A remarkable increased $\sigma / \tau$ is achieved with the increase of carrier concentration at different temperature. The $\sigma / \tau-n$ relationship reveals over two orders of magnitude improvement within the range of electron and hole concentrations we considered. For the p-type doped sample, the heavy doping level $\left(10^{21} \mathrm{~cm}^{-3}\right)$ can bring the same magnitude enhancement of $\sigma / \tau$, however, the value is significantly smaller than the n-type doping one. Generally, the electrical conductivity could be derived from the relation $\sigma=\operatorname{en} \mu$ ( $n$, carrier concentration; and $\mu$, carrier mobility). The remarkable disparity in conductivity value between two doping types is attributed to the high carrier mobility of n-type doping, related
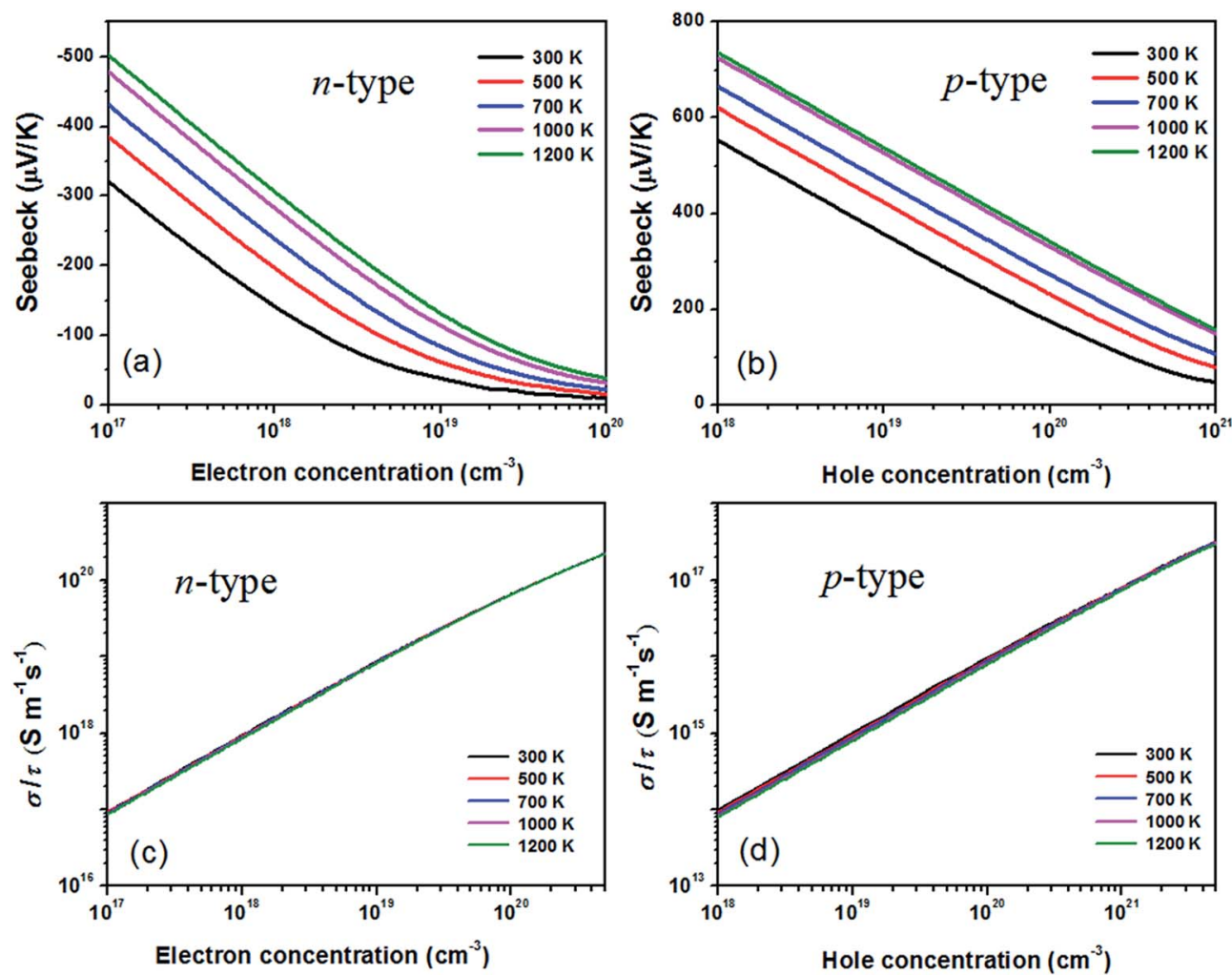

Fig. 2 The Seebeck coefficient (a) and (b), the ratio of $\sigma$ to $\tau$ (c) and (d) as a function of $\mathrm{n}$-type and p-type carrier concentration at different temperature. 
to the large dispersive conduction band. For p-type system, the high DOS effective mass due to flat valance band reduces the mobility although it yields high Seebeck coefficient. Therefore, the trade-off between electrical conductivity and Seebeck coefficient results in the peak power factor (PF) and $Z T$ value.

The constant relaxation time approximation is typically employed and the value of $\tau$ can be acquired by fitting it to the experimental results. Such method has been implemented to estimate the relaxation time in many other materials. ${ }^{47,48}$ With the available experimental electrical conductivities of n-type and p-type doped $\mathrm{BaSnO}_{3}$ adopted, ${ }^{17,20}$ we determine the relaxation times are $9.8 \times 10^{-15} \mathrm{~s}$ and $11.16 \times 10^{-16} \mathrm{~s}$ for $\mathrm{n}$-type and p-type system at room temperature, respectively. Furthermore, the electrical conductivities from experiment with different small carrier concentrations at room temperature give similar $\tau$ values, which indicates that $\tau$ is in weak dependence of the carrier concentration and the constant $\tau$ assumption is reasonable in small doping level. For the heavily doped samples, the temperature and carrier concentration dependence of $\tau$ is assumed in the typical electron-phonon form, $\tau=$ $A T^{-1} n^{-1 / 3}$ ( $\tau$ in s, $T$ in $\mathrm{K}, n$ in $\mathrm{cm}^{-3}$ ), where $A$ is a constant. With the experimental data, ${ }^{20}$ the yield $A$ is $2.94 \times 10^{-6} \mathrm{~s} \mathrm{~K} \mathrm{~cm}^{-1}$. With the calculated $\tau$, electrical conductivities as functions of carrier concentration are presented in Fig. 3(a) and (b). The $\sigma$ of n-type doping increases rapidly from $\sim 10^{3} \mathrm{~S} \mathrm{~m}^{-1}$ to $\sim 10^{6} \mathrm{~S} \mathrm{~m}^{-1}$ as the carrier concentration increase from $\sim 10^{17} \mathrm{~cm}^{-3}$ to $\sim 10^{20}$ $\mathrm{cm}^{-3}$, showing a transform from semiconductor to mental like behavior. The PF defined as $S^{2} \sigma$, is usually used as a criterion in searching for new thermoelectric materials with high output power. The significantly enhanced $\sigma$ satisfies the demonstration reported by Takada et al. ${ }^{49}$ that the improvement of the electrical conductivity of the $\mathrm{BaSnO}_{3}$ is very important to obtain a high power factor. The carrier concentration and temperature dependence of PF for n-type and p-type doping is shown in
Fig. 3(c) and (d). The value of maximum PF corresponds to the optimal carrier concentration, resulting from the opposite dependence on carrier concentration between $S$ and $\sigma$. Typically, the light electronic bands with small effective mass request low optimal carrier concentration $\left(10^{19}\right.$ to $\left.10^{20} \mathrm{~cm}^{-3}\right)$, namely, a low content of dopants is enough to optimize the power factor. In contrast, for the heavy band materials, higher carrier concentrations are demanded to optimize the power factor. Consequently, at $1200 \mathrm{~K}$, the PF values for n-type and ptype doped $\mathrm{BaSnO}_{3}$ reach the maximum at carrier concentration of $1.6 \times 10^{19} \mathrm{~cm}^{-3}$ and $2 \times 10^{21} \mathrm{~cm}^{-3}$, respectively. Considering the similar Seebeck coefficient but great disparity of electrical conductivity for n- and p-type doped $\mathrm{BaSnO}_{3}$ at optimal carrier concentration, the PF maximum of n-type is near four orders of magnitude higher than p-type one. Moreover, the PF increases with the rising of temperature. Thus a ultra-high optimal $\mathrm{PF}$ $\left(1.5 \times 10^{-3} \mathrm{~W} \mathrm{~m}^{-1} \mathrm{~K}^{-2}\right)$ of n-type doped $\mathrm{BaSnO}_{3}$ is obtained, which is standout amongst the oxide-based thermoelectric materials, such as $\mathrm{NaCo}_{2} \mathrm{O}_{4}$ (ref. 50) and the doped $\mathrm{CaMnO}_{3},{ }^{51}$ indicating the great potential for n-type doped $\mathrm{BaSnO}_{3}$ as a high temperature thermoelectric material. Our calculated PF values are in reasonable agreement with the experimental data. For example, at the carrier concentration of $7 \times 10^{19} \mathrm{~cm}^{-3}$ for n-type doped samples, the calculated value of $0.63 \times 10^{-3} \mathrm{~W} \mathrm{~m}^{-1} \mathrm{~K}^{-2}$ at $1000 \mathrm{~K}$ coincides with the experimented $0.45 \times 10^{-3} \mathrm{~W} \mathrm{~m}^{-1}$ $\mathrm{K}^{-2}$ at $973 \mathrm{~K}$. However, our simulated optimal PF value of $1.5 \times$ $10^{-3} \mathrm{~W} \mathrm{~m}^{-1} \mathrm{~K}^{-2}$ indicates the thermoelectric performance of $\mathrm{BaSnO}_{3}$ can be further improved and provides guidance of synergetic tuning optimum carrier concentration and higher temperature to experiment efforts.

Next, we investigate the thermal transport properties of $\mathrm{BaSnO}_{3}$. The electrical thermal conductivity $\kappa_{\mathrm{e}}$ is calculated from the electrical conductivity $\sigma$ via the Wiedemann-Franz relationship $\kappa_{\mathrm{e}}=L \sigma T$, where $L$ is the Lorenz number, which is
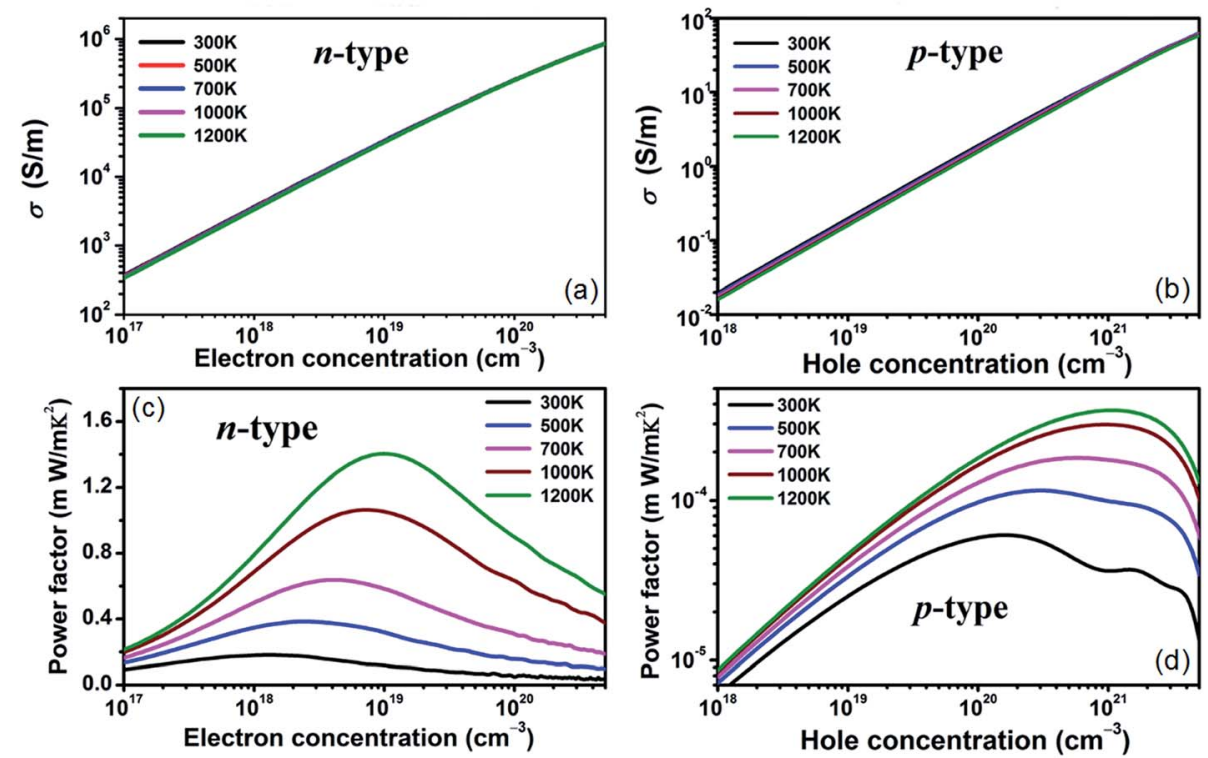

Fig. 3 Calculated electrical conductivity (a) and (b) and power factor (c) and (d) as a function of n-type and p-type carrier concentration at different temperature. 
approximately $2.44 \times 10^{-8} \mathrm{~W} \Omega \mathrm{K}^{-2}$. Consequently, the curves of $\kappa_{\mathrm{e}}$ versus carrier concentration exhibit the similar trends to $\sigma$, as shown in Fig. 4(a) and (b). In the degenerate semiconductor, the phonon conductivity is predominate part of the total thermal conductivity and thus the electronic contribution is negligible. The lattice thermal conductivity $\left(\kappa_{1}\right)$ and the total thermal conductivity ( $\kappa_{\text {total }}$ ) of the intrinsic $\mathrm{BaSnO}_{3}$ at different temperature are shown in Fig. 4(d). The $\kappa_{1}$ values estimated using ShengBTE code are slightly larger than the experimental value of $\kappa_{\text {total }}$, which originates from the following reason: in first principles approach only the phonon-phonon scattering is considered while in samples used for experimental measurement contain other types of scattering due to defects, impurities and dislocations which in turn reduce the thermal conductivity. The fitted line of $\kappa_{1}$ follows the $T^{-1}$ relationship, indicating the phonon-phonon interaction plays the main role in the phonon mechanism. The decreased $\kappa_{1}$ can be explained by the increased phonon-phonon scattering as temperature rising. To investigate the origin of the reduction of thermal conductivity, the phonon spectra of $\mathrm{BaSnO}_{3}$ is calculated and plotted in Fig. 4(c). The heavy constituent elements contribute mainly to the lower frequency mode, leading to the low frequency optical branches overlapping with the acoustic modes. Thus, the optical branches share part of heat with the acoustic branches, which increase the chance of phonon scattering. The experiment reported $\kappa_{\text {total }}$ decreases rapidly from $9.6 \mathrm{~W} \mathrm{~m}^{-1} \mathrm{~K}^{-1}$ to $3.2 \mathrm{~W} \mathrm{~m}^{-1}$ $\mathrm{K}^{-1}$ as temperature increases from $323 \mathrm{~K}$ to $1263 \mathrm{~K} .^{29}$ Compared to the reported thermal conductivity of n-type doped
$\mathrm{Ba}_{1-x} \mathrm{La}_{x} \mathrm{SnO}_{3}$, the thermal conductivities are quite similar at different doping levels, ${ }^{52}$ indicating the $\kappa_{\text {total }}$ is independent with carrier concentration. As to the doped compound, the electronic contribution, $\kappa_{\mathrm{e}}$ is gradually enhanced due to the positive relationship to the carrier concentration. However, in thermoelectric materials, dopants not only supply carriers to optimize the power factor, but enhance the phonon scattering to reduce the contribution of $\kappa_{1}$. Thus, the compromise between $\kappa_{\mathrm{e}}$ and $\kappa_{\mathrm{l}}$ contributes to the doping level independence of $\kappa_{\text {total }}$.

With the electronic and thermal transport coefficients available, we now evaluate the thermoelectric performance of $\mathrm{BaSnO}_{3}$ by $Z T$ value and conversion efficiency. Considering the small power factor value of p-type doping is meaningless to practical application, only the $Z T$ of n-type doped $\mathrm{BaSnO}_{3}$ as a function of carrier concentration at different temperature is shown in Fig. 5. At low temperature $(T<700 \mathrm{~K})$, the $Z T$ is almost independent with the carrier concentration, possessing small values. However, there is a remarkable increase when the temperature elevates beyond $700 \mathrm{~K}$. The $Z T$ as a function of carrier concentration is in similar tendency to that of power factor. The maximal $Z T$ value of 0.65 is obtained with the optimal electron doping concentration of $1.6 \times 10^{19}$ $\mathrm{cm}^{-3}$ at $1200 \mathrm{~K}$, which is higher than other well-known oxide thermoelectric materials in solid states. ${ }^{53}$ The improved $Z T$ value is originated from the significantly increased electrical conductivity with n-type doping as well as the high temperature stability. As known, in thermoelectric device application, not only a high $Z T$ peak but the high $Z T$ value over a wide
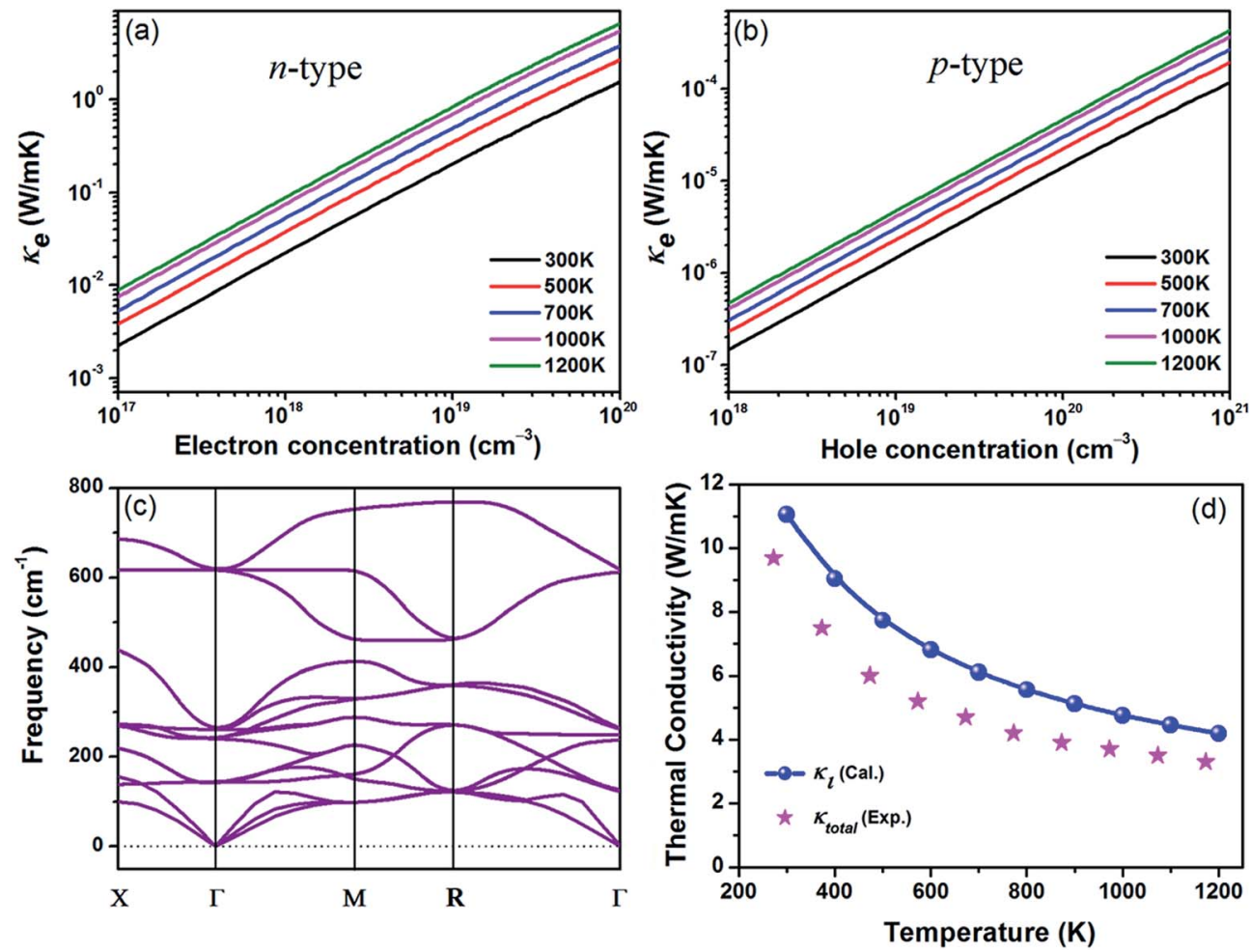

Fig. 4 Electronic thermal conductivity as a function of carrier concentration at different temperature for $n$-type (a) and p-type (b) doped $\mathrm{BaSnO}$. (c) Phonon dispersion of pure $\mathrm{BaSnO}_{3}$. (d) Lattice thermal conductivity and total thermal conductivity. 


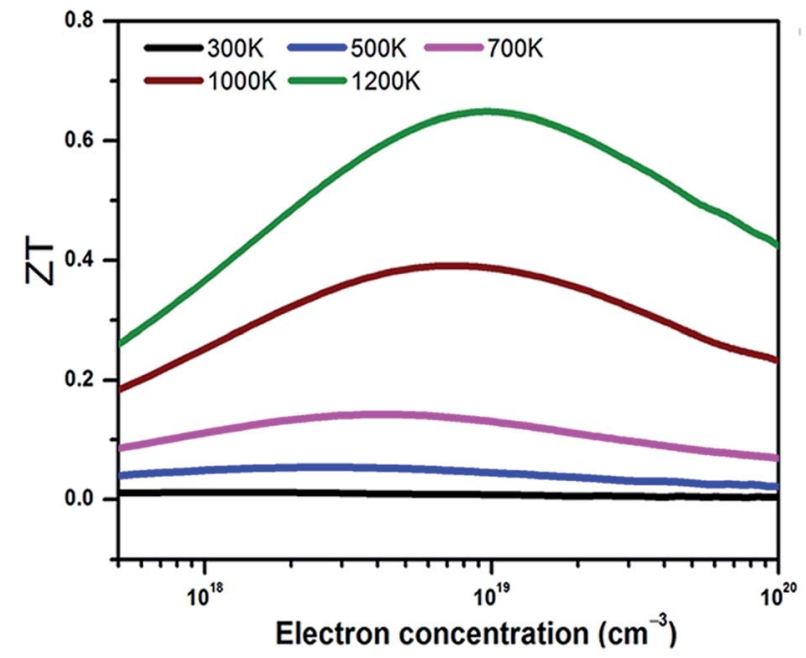

Fig. 5 The $Z T$ value of n-type doped $\mathrm{BaSnO}_{3}$ single crystal as a function of carrier concentration at different temperature.

temperature range is required..$^{54}$ The average $Z T$ value of $\mathrm{n}$ type doped $\mathrm{BaSnO}_{3}$ is calculated to be $\sim 0.33$ and $\sim 0.52$ in the temperature of $300-1200 \mathrm{~K}$ and $900-1200 \mathrm{~K}$, respectively. It is deduced that optimizing the $Z T$ value by tuning the carrier concentration at high temperature makes $\mathrm{BaSnO}_{3}$ to be a promising alternative in the great demanding for $n$-type thermoelectric materials nowadays. The main factor that degrade the $Z T$ value at low temperature is the large thermal conductivity. Creating strong point-defect scattering by introducing dopants and enhancing phonon-phonon scattering by nanostructuring are effective ways to suppress the thermal conductivity. Furthermore, the quantum confinement effect of electronic carriers in the low-dimension can significantly enhance the thermo power. We predict the $Z T$ value could be further boosted in the nanostructured $\mathrm{BaSnO}_{3}$.

In practical application of thermoelectric device, the energy conversion efficiency is the direct index to assess the thermoelectric performance. The ideal maximum efficiency $(\eta)$ characterizes the heat-electricity conversion ability of thermoelectric modules, which can be presented using the equation, ${ }^{55}$

$$
\eta=\eta_{\mathrm{c}} \frac{\sqrt{1+\overline{Z T}}-1}{\sqrt{1+\overline{Z T}}+T_{\mathrm{c}} / T_{\mathrm{h}}}
$$

where, $T_{\mathrm{c}}$ and $T_{\mathrm{h}}$ represent the temperature of cold side and hot resource of thermoelectric device. $\eta_{\mathrm{c}}=\left(T_{\mathrm{h}}-T_{\mathrm{c}}\right) / T_{\mathrm{c}}$ is the Carnot efficiency. The dimensionless $\overline{Z T}$ is the average figure of merit between the hot side and cold side thermoelectric materials of the module. To examine the practicability of $\mathrm{BaSnO}_{3}$ in thermoelectric devices, the modules based on the n-type $\mathrm{BaSnO}_{3}$ and four popular p-type materials are assembled (see Fig. S1 of ESI $\dagger$ ). The high maximum conversion efficiency based on n-type $\mathrm{BaSnO}_{3}$ and p-type half-Heusler FeNbSb under a high temperature difference of $900 \mathrm{~K}$ is achieved, suggesting the great potential of n-type $\mathrm{BaSnO}_{3}$ for high temperature power generation.

\section{Conclusions}

We systematically investigated the geometric, electronic and thermoelectric properties of perovskite-type $\mathrm{BaSnO}_{3}$ by the firstprinciple calculations combined with the semi-classical Boltzmann theory. The comparison of thermoelectric properties between n-type and p-type doped $\mathrm{BaSnO}_{3}$ were performed. The results revel that the n-type doped system displays an ultra-high power factor of $1.5 \times 10^{-3} \mathrm{~W} \mathrm{~m}^{-1} \mathrm{~K}^{-2}$ at $1200 \mathrm{~K}$ with optimal carrier concentration of $1.6 \times 10^{19} \mathrm{~cm}^{-3}$. The excellent $\mathrm{PF}$ is attributed to the dramatically increased electrical conductivity, originating from the small effective mass of electron and extraordinary high mobility. The enhanced $\mathrm{PF}$ and $Z T$ values serve as guides to related experiments in pointing out the importance of doping levels and operation temperature to optimize the thermoelectric performance of $\mathrm{BaSnO}_{3}$. The lattice thermal conductivity can be suppressed by increasing temperature and introducing dopants. In addition, four types of thermoelectric modules formed by n-type $\mathrm{BaSnO}_{3}$ and p-type thermoelectric materials were assembled.

\section{Acknowledgements}

This work was supported by the National Natural Science Foundation of China (No. 21501177). We acknowledge the Supercomputing Center of Shenzhen and CNIC for providing the computer resources.

\section{References}

1 B. Poudel, Q. Hao, Y. Ma, Y. C. Lan, A. Minnich, B. Yu, X. Yan, D. Z. Wang, A. Muto, D. Vashaee, X. Y. Chen, J. M. Liu, M. S. Dresselhaus, G. Chen and Z. F. Ren, Science, 2008, 320, 634-638.

2 L. D. Zhao, S. H. Lo, Y. S. Zhang, H. Sun, G. J. Tan, C. Uher, C. Wolverton, V. P. Dravid and M. G. Kanatzidis, Nature, 2014, 508, 373-377.

3 J. P. Heremans, V. Jovovic, E. S. Toberer, A. Saramat, K. Kurosaki, A. Charoenphakdee, S. Yamanaka and G. J. Snyder, Science, 2008, 321, 554-557.

4 Y. Z. Pei, X. Y. Shi, A. LaLonde, H. Wang, L. D. Chen and G. J. Snyder, Nature, 2011, 473, 66-69.

5 Y. B. Luo, J. Y. Yang, M. Liu, Y. Xiao, L. W. Fu, W. X. Li, D. Zhang, M. Y. Zhang and Y. D. Cheng, J. Mater. Chem. A, 2015, 3, 1251-1257.

6 J. Li, Z. Ma and K. Wu, RSC Adv., 2017, 7, 8460-8466.

7 G. J. Snyder and E. S. Toberer, Nat. Mater., 2008, 7, 105-114.

8 K. Koumoto, Y. F. Wang, R. Z. Zhang, A. Kosuga and

R. Funahashi, Annu. Rev. Mater. Res., 2010, 40, 363-394.

9 G. K. Ren, J. L. Lan, C. C. Zeng, Y. C. Liu, B. Zhan, S. Butt, Y. H. Lin and C. W. Nan, JOM, 2015, 67, 211-221.

10 M. Lee, L. Viciu, L. Li, Y. Wang, M. L. Foo, S. Watauchi, R. A. Pascal Jr, R. J. Cava and N. P. Ong, Nat. Mater., 2006, 5, 537-540.

11 J. Sugiyama, J. H. Brewer, E. J. Ansaldo, H. Itahara, T. Tani, M. Mikami, Y. Mori, T. Sasaki, S. Hebert and A. Maignan, Phys. Rev. Lett., 2004, 92, 017602. 
12 Y. Masuda, D. Nagahama, H. Itahara, T. Tani, W. S. Seo and K. Koumoto, J. Mater. Chem., 2003, 13, 1094-1099.

13 H. Ohta, K. Sugiura and K. Koumoto, Inorg. Chem., 2008, 47, 8429-8436.

14 Y. Liu, J. L. Lan, W. Xu, Y. C. Liu, Y. L. Pei, B. Cheng, D. B. Liu, Y. H. Lin and L. D. Zhao, Chem. Commun., 2013, 49, 8075-8077.

15 L. D. Zhao, J. Q. He, D. Berardan, Y. H. Lin, J. F. Li, C. W. Nan and N. Dragoe, Energy Environ. Sci., 2014, 7, 2900-2924.

16 H. Ohta, S. Kim, Y. Mune, T. Mizoguchi, K. Nomura, S. Ohta, T. Nomura, Y. Nakanishi, Y. Ikuhara, M. Hirano, H. Hosono and K. Koumoto, Nat. Mater., 2007, 6, 129-134.

17 W. S. Choi, H. K. Yoo and H. Ohta, Adv. Funct. Mater., 2015, 25, 799-804.

18 T. Tsubota, M. Ohtaki, K. Eguchi and H. Arai, J. Mater. Chem., 1997, 7, 85-90.

19 A. M. Dehkordi, S. Bhattacharya, T. Darroudi, J. W. Graff, U. Schwingenschloegl, H. N. Alshareef and T. M. Tritt, Chem. Mater., 2014, 26, 2478-2485.

20 H. J. Kim, U. Kim, T. H. Kim, J. Kim, H. M. Kim, B. G. Jeon, W. J. Lee, H. S. Mun, K. T. Hong, J. Yu, K. Char and K. H. Kim, Phys. Rev. B: Condens. Matter Mater. Phys., 2012, 86, 165205.

21 H. J. Kim, U. Kim, H. M. Kim, T. H. Kim, H. S. Mun, B. G. Jeon, K. T. Hong, W. J. Lee, C. Ju, K. H. Kim and K. Char, Appl. Phys. Express, 2012, 5, 061102.

22 X. Luo, Y. S. Oh, A. Sirenko, P. Gao, T. Tyson, K. Char and S. W. Cheong, Appl. Phys. Lett., 2012, 100, 172112.

23 H. Mizoguchi, P. Chen, P. Boolchand, V. Ksenofontov, C. Felser, P. W. Barnes and P. M. Woodward, Chem. Mater., 2013, 25, 3858-3866.

24 H. R. Liu, J. H. Yang, H. J. Xiang, X. G. Gong and S. H. Wei, Appl. Phys. Lett., 2013, 102, 112109.

25 D. O. Scanlon, Phys. Rev. B: Condens. Matter Mater. Phys., 2013, 87, 161201.

26 H. J. Kim, J. Kim, T. H. Kim, W. J. Lee, B. G. Jeon, J. Y. Park, W. S. Choi, D. W. Jeong, S. H. Lee, J. Yu, T. W. Noh and K. H. Kim, Phys. Rev. B: Condens. Matter Mater. Phys., 2013, 88, 125204.

27 B. Ostrick, M. Fleischer, U. Lampe and H. Meixner, Sens. Actuators, B, 1997, 44, 601-606.

28 A. Bouhemadou and K. Haddadi, Solid State Sci., 2010, 12, 630-636.

29 T. Maekawa, K. Kurosaki and S. Yamanaka, J. Alloys Compd., 2006, 416, 214-217.

30 S. Ohta, T. Nomura, H. Ohta and K. Koumoto, J. Appl. Phys., 2005, 97, 034106.

31 K. Takahata, Y. Iguchi, D. Tanaka, T. Itoh and I. Terasaki, Phys. Rev. B: Condens. Matter Mater. Phys., 2000, 61, 12551.

32 F. Li, J. F. Li, L. D. Zhao, K. Xiang, Y. Liu, B. P. Zhang, Y. H. Lin, C. W. Nan and H. M. Zhu, Energy Environ. Sci., 2012, 5, 7188-7195.
33 M. Scheele, N. Oeschler, K. Meier, A. Kornowski, C. Klinke and H. Weller, Adv. Funct. Mater., 2009, 19, 3476-3483.

34 L. D. Zhao, G. J. Tan, S. Q. Hao, J. Q. He, Y. L. Pei, H. Chi, H. Wang, S. K. Gong, H. B. Xu, V. P. Dravid, C. Uher, G. J. Snyder, C. Wolverton and M. G. Kanatzidis, Science, 2016, 351, 141-144.

35 P. E. Blöchl, Phys. Rev. B: Condens. Matter Mater. Phys., 1994, 50, 17953-17979.

36 G. Kresse and J. Furthmüller, Phys. Rev. B: Condens. Matter Mater. Phys., 1996, 54, 11169-11186.

37 J. Heyd, G. E. Scuseria and M. Ernzerhof, J. Chem. Phys., 2003, 118, 8207-8215.

38 G. K. H. Madsen and D. J. Singh, Comput. Phys. Commun., 2006, 175, 67-71.

39 A. Togo, F. Oba and I. Tanaka, Phys. Rev. B: Condens. Matter Mater. Phys., 2008, 78, 134106.

40 W. Li, J. Carrete, N. A. Katcho and N. Mingo, Comput. Phys. Commun., 2014, 185, 1747-1758.

41 E. H. Mountstevens, J. P. Attfield and S. A. Redfern, J. Phys.: Condens. Matter, 2003, 15, 8315-8326.

42 H. Mizoguchi, H. W. Eng and P. M. Woodward, Inorg. Chem., 2004, 43, 1667-1680.

43 H. Mizoguchi, P. M. Woodward, C. H. Park and D. A. Keszler, J. Am. Chem. Soc., 2004, 126, 9796-9800.

44 D. Singh, D. Papaconstantopoulos, J. Julien and F. CyrotLackmann, Phys. Rev. B: Condens. Matter Mater. Phys., 1991, 44, 9519-9523.

45 É. Bévillon, A. Chesnaud, Y. Wang, G. Dezanneau and G. Geneste, J. Phys.: Condens. Matter, 2008, 20, 145217.

46 T. N. Stanislavchuk, A. A. Sirenko, A. P. Litvinchuk, X. Luo and S. W. Cheong, J. Appl. Phys., 2012, 112, 044108.

47 G. K. H. Madsen, J. Am. Chem. Soc., 2006, 128, 12140-12146.

48 A. N. Gandi and U. Schwingenschloegl, Chem. Mater., 2014, 26, 6628-6637.

49 M. Takada, H. Taguchi, M. Kato and K. Hirota, J. Mater. Sci., 2015, 50, 476-481.

50 I. Terasaki, Y. Sasago and K. Uchinokura, Phys. Rev. B: Condens. Matter Mater. Phys., 1997, 56, 685-687.

51 L. Bocher, M. Aguirre, D. Logvinovich, A. Shkabko, R. Robert, M. Trottmann and A. Weidenkaff, Inorg. Chem., 2008, 47, 8077-8085.

52 M. Yasukawa, T. Kono, K. Ueda, H. Yanagi, S. W. Kim and H. Hosono, Solid State Commun., 2013, 172, 49-53.

53 E. Guilmeau, P. Diaz-Chao, O. I. Lebedev, A. Recnik, M. C. Schafer, F. Delorme, F. Giovannelli, M. Kosir and S. Bernik, Inorg. Chem., 2017, 56, 480-487.

54 L. Zhao, G. Tan, S. Hao, J. He, Y. Pei, H. Chi, H. Wang, S. Gong, H. Xu and V. P. Dravid, Science, 2016, 141-144.

55 D. Kraemer and G. Chen, Rev. Sci. Instrum., 2014, 85, 045107. 